\title{
Sitios utilizados por la nutria neotropical en una selva baja caducifolia en la costa de Oaxaca, México
}

Ma. Antonieta Casariego-Madorell ${ }^{*}$

\section{Abstract}

This work was carried out in the river Copalita and Zimatán located in Huatulco within the municipalities of San Pedro Huamelula, Santiago Astata and San Miguel del Puerto in Oaxaca State. The samplings were carried out July 1999 to September 2000, identifying key areas for otters, such as resting sites and sites frequently used for sprainting. This was done by walking down the river banks, looking for any evidence of the presence of otters, such as footprints, food remains and scats, all of them were recorded. Two dens were found on Zimatán; this river was apparently preferred Copalita River, although no significant differences in frequency of use were found between these rivers. There were other organism recorded, such as raccoons, skunks, foxes, jaguaroundi, puma and jaguar. Results emphasizes the importance of protecting these areas, not only for the otter, but also for the others species that are found in this area.

Key words: Dens, frequency of use, Neotropical otter, Oaxaca, scats.

\section{Resumen}

Este trabajo se realizó en los ríos Copalita y Zimatán ubicados en Huatulco dentro de los municipios de San Pedro Huamelula, Santiago Astata y San Miguel del Puerto en el estado de Oaxaca. Los muestreos se llevaron a cabo del mes de julio de 1999 al mes de septiembre del 2000, determinando las áreas importantes para la nutria como lo son sitios de descanso y los sitios frecuentes destinados al marcaje, esto se llevó a cabo recorriendo las orillas de los ríos a pie buscando cualquier evidencia de la presencia de la nutria, como huellas, restos de alimento y excretas las cuales fueron contabilizadas. Se encontraron dos madrigueras y preferencia por el río Zimatán, no se encontraron diferencias significativas en la frecuencia de uso con el río Copalita. También se registraron otros organismos como mapaches, zorrillos, zorras, jaguaroundi, puma y jaguar. Lo que destaca la importancia de la protección de estas zonas no únicamente por la nutria sino también por todas las especies que ahí cohabitan.

Palabras clave: Excretas, frecuencia de uso, madrigueras, nutria neotropical, Oaxaca.

Instituto de Ecología, Universidad Nacional Autónoma de México, Apartado Postal 70-275, 04510 México D. F., México. Email: madorell@prodigy.net.mx.

*Corresponding author 
neotropical necesarios para asegurar la permanencia de las poblaciones de nutria en En la actualidad en América Latina han aumentado de manera considerable los trabajos sobre la nutria neotropical Lontra longicaudis annectens, encaminados a su protección debido a que se encuentra incluida en el Apéndice I de la Convención Internacional para el Tráfico de Especies de Flora y Fauna Amenazadas (CITES). En México es considerada como amenazada por la NOM-059-ECOL-2010 (SEMARNAT 2010) debido a que sus poblaciones han sido seriamente diezmadas como en Argentina y Costa Rica, por factores como la cacería, la destrucción de su hábitat y/o la contaminación de las aguas (Gallo-Reynoso 1986; Alho et al. 1988; Chehébar 1991). Estos estudios en su mayoría se han enfocado principalmente a cuestiones de descripción, alimentación, distribución y tamaño de sus poblaciones (Melquist 1983; Melquist y Dronkert 1987; Parera 1992, 1996; Spinola y Vaughan 1995; Helder y De Andrade 1997; Quadros y Monteiro-Filho 2002; Gori et al. 2003). Destacando en México sobre la misma línea de investigación los trabajos de Gallo-Reynoso (1989, 1996), Macías-Sánchez y Aranda (1999), OrozcoMeyer (1998), Macías-Sánchez (2003), Casariego-Madorell et al. (2008). Sin embargo, aún se sabe poco sobre la relación de estos organismos con su medio ambiente. Con respecto a los factores que influyen en la presencia en los diversos sitios donde habita, se sabe que existe una alta selección positiva de los espacios conservados y una selección negativa de los espacios más alterados, considerando como principales elementos para su establecimiento el desarrollo de la vegetación y la disponibilidad de alimento (LópezMartín et al. 1998). Uno de los aspectos un tanto descuidado en estas investigaciones son los lugares que visita la nutria frecuentemente tal es el caso de los lugares de descanso (Quadros y Monteiro-Filho 2002). Los cuales son aquellos lugares que utiliza regularmente la nutria, generalmente cerca del agua, entre las raíces de los árboles o en cavidades de las rocas, siendo lugares apropiados para poder construir madrigueras y en condiciones ideales son los ríos flanqueados de grandes bloques rocosos (Pardini y Trajano 1999), lugares que también utilizan para marcar o donde depositar sus excretas.

La presencia de la nutria es considerada como bioindicador en la evaluación de la calidad de diversos ambientes acuáticos (Staib y Schenck 1994), su conservación es importante porque es una especie amenazada debido a que posee características intrínsecas que la hacen especialmente vulnerable a las reducciones poblacionales. La nutria neotropical es una especie que necesita amplios espacios para vivir, por lo que proteger las superficies requeridas por las poblaciones de la nutria, también se protege a otros organismo presentes en dichas áreas (Hernández 1992).

La nutria neotropical se encuentra en áreas templadas y tropicales desde el noroeste de México hasta Argentina (Gallo-Reynoso 1996; Aranda 2000), en México el bosque tropical caducifolio o selva baja caducifolia es el ecosistema más amenazado de los que ocupa la nutria (Janzen 1988; Ceballos y García 1995). En el estado de Oaxaca se mantienen franjas continuas de bosque tropical caducifolio. En las cercanías del río Zimatán existen algunos fragmentos aislados (García et al. 1992; Salas-Morales 2002), junto con el río Copalita y el río Ayuta, son los ríos más importantes en el área de Huatulco en los municipios de San Pedro Huamelula, Santiago Astata y San Miguel del Puerto (Salas et al. 1996). Estos ríos al ser perennes y por presentar caudales rápidos representan una oportunidad para realizar estudios de ecología sobre la nutria Material

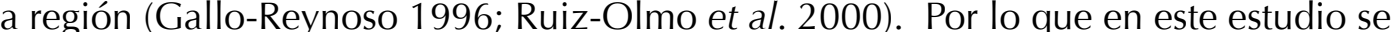
y Métodos

Área de estudio. Los ríos Copalita y Zimatán se encuentran en la costa de Oaxaca, entre los $15.717^{\circ}$ y $16.833^{\circ} \mathrm{N}$ y $-95.750^{\circ}$ y $-96.250^{\circ} \mathrm{W}$ y dentro de los municipios de San Pedro Huamelula, Santiago Astata y San Miguel del Puerto (Fig. 1).
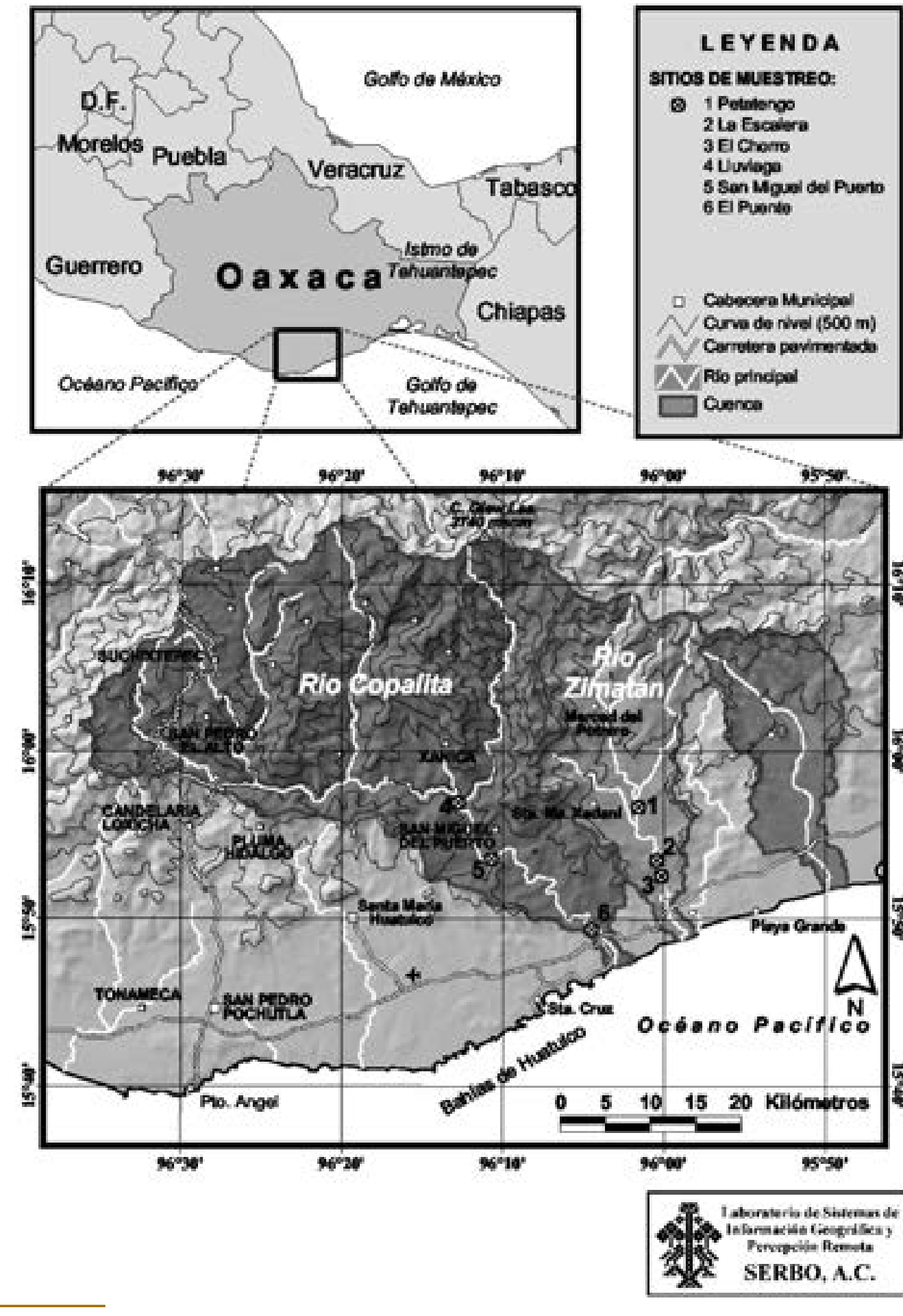

96w

$95 \times$ 
El área de estudio comprende una superficie de 152,535 ha para el río Copalita y 46,081 ha para el río Zimatán. Estos ríos son importantes para las nutrias ya que son corrientes permanentes en esta costa. El clima es cálido subhúmedo (Aw; según Köeppen modificado por García 1978), con una temperatura media anual de $28^{\circ} \mathrm{C}$ y una precipitación promedio anual de 700 a $800 \mathrm{~mm}$. Se caracteriza por presentar una estacionalidad con dos temporadas bien definidas: la de lluvias de mayo a octubre, y la de secas de noviembre a abril (INEGI 1988). Los principales tipos de vegetación son el bosque tropical caducifolio o selva baja; la selva tropical subcaducifolia a la orilla de los ríos; la selva tropical subperenifolia hacia la porción norte en los sitios cercanos a las serranías, y en los alrededores de las lagunas costeras, franjas angostas de manglar (Rzedowski 1978; Salas-Morales 2002).

Para cada uno de los ríos se determinaron las especies de flora presentes en los alrededores y se consideraron las más abundantes para la zona. Para obtener la altura de las rocas fue por observación con puntos de referencia y cuando era posible medidas con un metro (Truper®). El sustrato de la zona se obtuvo de acuerdo a Franco-López et al (1985). Las características fisicoquímicas que se tomaron en cuenta fueron; temperatura ambiental $\left({ }^{\circ} \mathrm{C}\right)$ temperatura del agua $\left({ }^{\circ} \mathrm{C}\right)$; lecho (ancho real del río), cauce (área que ocupa el agua dentro del lecho), la profundidad, la velocidad del agua, la turbiedad, el oxígeno disuelto en el agua, alcalinidad y el pH (Lloyd 1992). Para obtener el ancho total del río, el cauce y la profundidad se utilizó un metro. La temperatura tanto ambiental como del agua se obtuvieron con un termómetro de mercurio $\left(\right.$ Brannan $\left.{ }^{\circledR}\right)$. La obtención del oxígeno disuelto y la alcalinidad se realizaron de acuerdo a la metodología sugerida por Franco-López et al (1985). Los reactivos y el material utilizados para llevar a cabo las pruebas fueron proporcionados por el laboratorio de botánica de la FES Iztacala. La turbiedad y la velocidad del agua fueron obtenidas de acuerdo a Contreras (1994). El pH se obtuvo con papel indicador (Merk®). Los muestreos se realizaron durante los meses de abril a agosto del 2000. Para cada una de las pruebas se obtuvieron 135 muestras por río (Casariego-Madorell 2004).

El río Copalita abarca tres diferentes poblados; Yuviaga en la parte alta, en el centro el poblado de San Miguel del Puerto, y en la parte baja Copalita. Este río es el más largo de la zona. Su cuenca ha sido alterada, por lo que durante la época de lluvia, el agua que lleva es turbia de color café intenso, lo que no se observa en los otros ríos. Entre las especies más abundantes de flora a la orilla del río se encuentran miembros de la familia Bignoniaceae como Astianthus viminalis y de la familia Leguminosae como Andira inermis y Albizia occidentalis. Encontrando también ejemplares de Pithecellobium dulce, Mazuma ulmifolia, Tabebuia sp., y Ceiba pentandra. No es común encontrar rocas mayores de $2 \mathrm{~m}$ (Fig. 2). Aunque hay una pared de aproximadamente $5 \mathrm{~m}$ de altura.

De acuerdo con las características fisicoquímicas se observa que es un río con zonas profundas y corrientes rápidas; puede llegar a tener más del $50 \%$ de turbiedad durante la época de lluvia, manteniendo una concentración media de oxígeno disuelto en el agua de $3.4 \pm 0.2 \mathrm{mg} / \mathrm{l}$, con un $\mathrm{pH}$ de $6-7$; con una temperatura promedio en el ambiente de $29^{\circ} \mathrm{C}$ y una temperatura promedio de $24.4^{\circ} \mathrm{C}$ en el agua. En este río se práctica el recorrido en kayak, ecoturismo y la pesca de subsistencia. Principalmente se tienen sembradíos de café en las partes altas del río, y de papaya y toronja a la orilla de algunos tramos del río, cerca de la bocana se extrae material para construcción constituyendo un área altamente perturbada (Casariego-Madorell et al. 2008).

El río Zimatán pertenece a la cuenca del mismo nombre, la cual presenta un gran número de brazos que se extienden en toda el área. Las especies más abundantes de flora son las mismas que en el río Copalita, aunque también se encontraron cactáceas de los géneros Mammillaria, Neobuxbaumia y Stenocereus, así como diversas especies del género Acacia y Mimosa. En esta zona se encuentran peñascos de hasta $5 \mathrm{~m}$ de altura donde se observa escasa vegetación, la cual en su mayoría son cactáceas. Se encuentran también áreas de arena y grava con extensa cobertura vegetal (Fig. 3). De acuerdo a las características fisicoquímicas este es un río en su mayoría profundo con corrientes rápidas y pozas de profundidad media entre los 80 y $100 \mathrm{~cm}$, la turbiedad nunca fue de más de $20 \%$, y el oxígeno disuelto en el agua es de $6.1 \pm 0.3 \mathrm{mg} / \mathrm{l} \mathrm{y} \mathrm{un} \mathrm{pH}$ de 7 . Con una temperatura promedio en el ambiente de $30.2{ }^{\circ} \mathrm{C}$ y una temperatura promedio de $26.2^{\circ} \mathrm{C}$ en el agua. En este río se ha observado el ecoturismo sobre todo en la parte baja llamada "el Chorro"; también se puede encontrar ganado, aunque la mayoría de los habitantes practican la cacería de subsistencia y en menor proporción para la venta (Alfaro-Espinoza 2000; Casariego-Madorell et al. 2008).

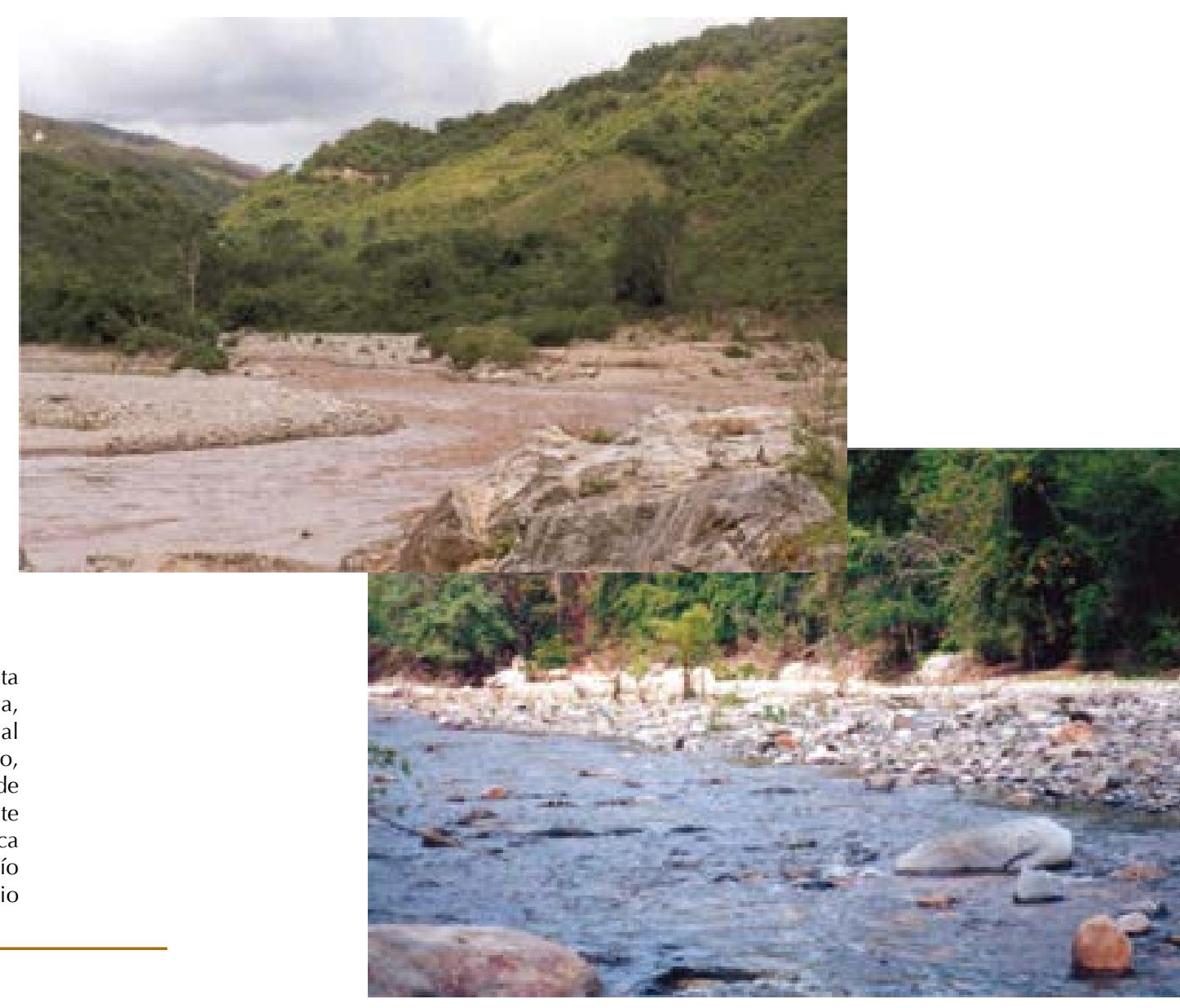

Obtención de las muestras: Para cada uno de los ríos se definieron tres transectos de 2 $\mathrm{km}$ de longitud. Cada transecto incluyó una franja de $5 \mathrm{~m}$ de ancho desde la orilla hacia el interior del bosque (Ruiz-Olmo et al. 1998). El recorrido de los transectos abarcó
Figura 2. Río Copalita de origen terrígen así como zonas arena (arriba) y dur dere de lluvia, cuando el se puede apreciar limpio abajo). 
15 meses de julio de 1999 a septiembre del 2000, los transectos se hicieron a pie. Las salidas se realizaron cada mes con una duración de siete días para el recorrido de ambos ríos, buscando los posibles sitios de descanso, de marcaje y/o madrigueras con la presencia de huellas, excretas, restos de alimento y marcas de garras entre otros (GalloReynoso 1989; Aranda 2000). Cuando fueron encontrados pelos, éstos se analizaron comparando la medula con los que se obtuvieron como referencia de los mamíferos en la zona para saber si se alimentaba de otros mamíferos (Arita y Aranda 1987).

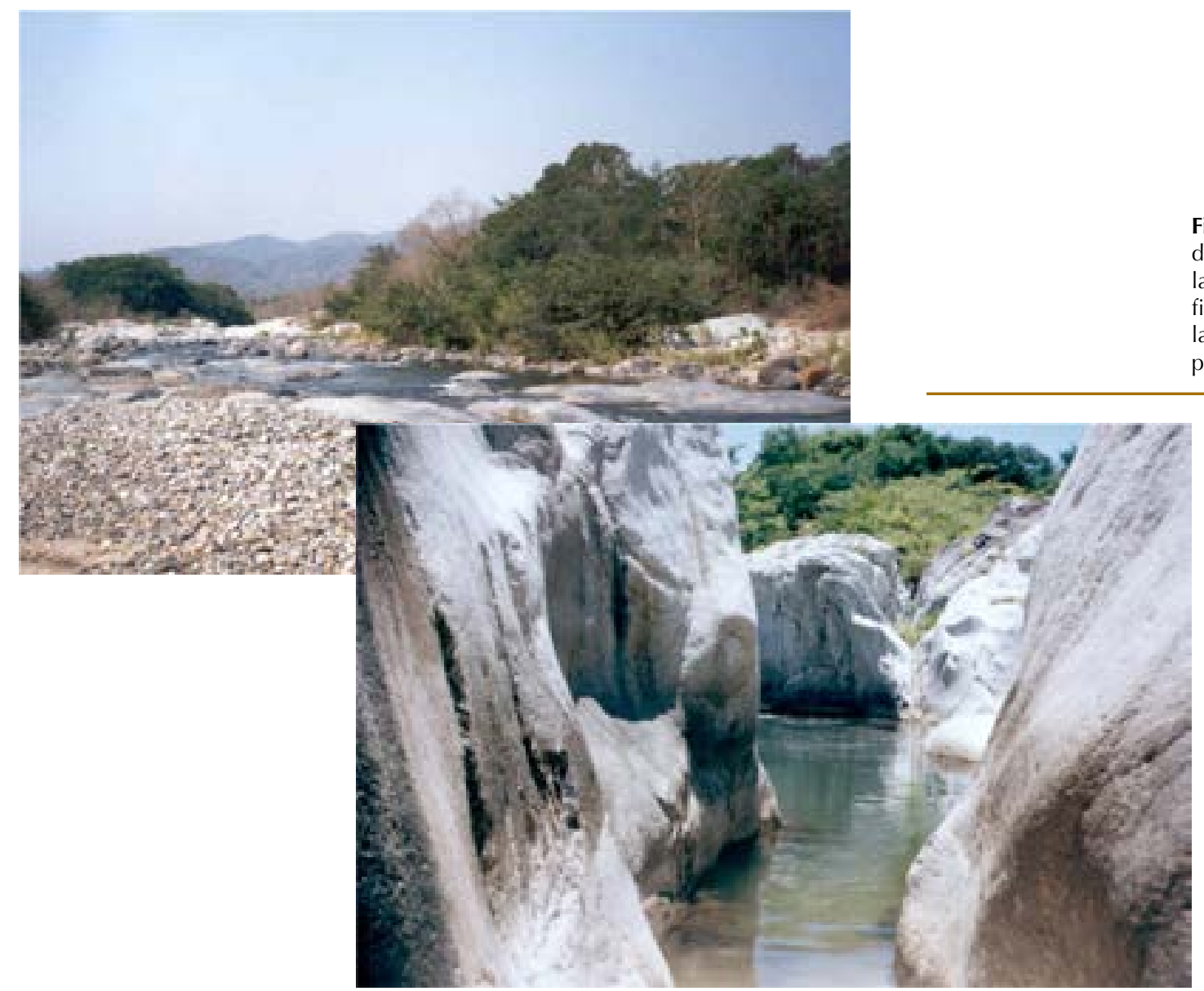

Se contabilizaron todas las excretas, incluyendo aquellas que fueron recolectadas y procesadas para obtener el tamaño poblacional y alimentación de la nutria en una investigación previa (Casariego-Madorell et al. 2008). Se tomaron en cuenta como sitios utilizados aquellos donde se encontraron diferentes tipos de rastros. Se consideró la posición de la excreta sobre el río y el sustrato en donde fueron encontradas. Al mismo tiempo se buscaron rastros de otros mamíferos, los cuales fueron determinados hasta nivel de especie cuando fue posible.

Análisis de datos. Para determinar si existían diferencias significativas entre los sitios frecuentes en ambos ríos se aplicó la prueba no paramétrica de Kruskal-Wallis y para determinar si existían diferencias de uso entre los ríos Copalita y Zimatán se optó por la prueba de Chi cuadrada (Zar 1999). Se consideró la frecuencia de uso de cada sitio calculando el número de meses con al menos una señal de rastro sobre el total de meses visitados de acuerdo al criterio de Quadros y Monteiro-Filho (2002).
Resultados Sigura 3. Río Zimatán, zona de arena y grava as paredes, así como las pozas (abajo).

Figura 4. Excretas $/ \mathrm{km}$ obtenidas en los río desde julio de 1999 a agosto del 2000 .
Se tomaron en cuenta un total de 802 excretas tanto observadas como colectadas, 186 para el río Copalita y 616 para el río Zimatán. Encontrando 2.06 excretas $/ \mathrm{km}$ y 6.84 excretas $/ \mathrm{km}$ en cada río respectivamente. En Copalita durante el mes de enero (temporada seca) se obtuvo el mayor número de excretas, mientras que el menor número de excretas se registró en los meses de julio, agosto, octubre y mayo de 1999, así como agosto del 2000 (temporada de lluvia). Para el río Zimatán, el mayor número de excretas se encontró en el mes de marzo (temporada seca), y el menor número de excretas se registró durante los meses de julio, agosto y septiembre de 1999, y febrero y agosto del 2000 (temporada de lluvia; Fig. 4). En ambos ríos se obtuvo el mayor número de excretas durante la temporada de seca. No se encontraron diferencias significativas entre el número de excretas $/ \mathrm{km}$ encontradas en el río Copalita y el río Zimatán $\left(X^{2}=2.57\right.$, g. I. $=1, P=0.11$.

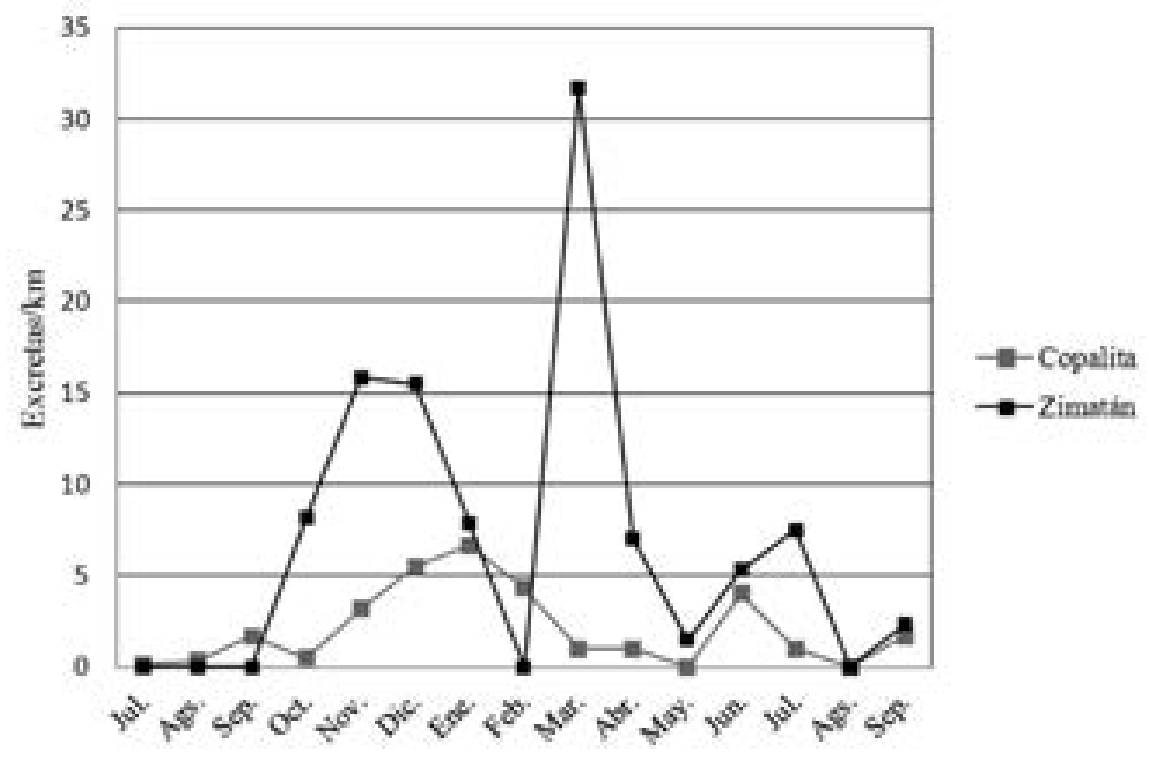

Los sitios considerados como de uso frecuente fueron las rocas a la orilla del río posteriormente las isletas, seguidos por los sitios en la ribera y finalmente troncos caídos también en la ribera (Tabla 1). Se obtuvo que para el río Copalita se tiene una frecuencia de ocurrencia de $0.48 \pm 0.004,48 \%$ incluyendo los tres transectos, en tanto que en el río Zimatán se tiene una frecuencia de ocurrencia de $0.51 \pm 0.04,51 \%$ para los tres transectos. No se encontraron diferencias significativas entre los tres sitios de uso del río Copalita $(H=1.12$, g. $\mathrm{l} .=2, P>0.05)$ ni entre los tres sitios del río Zimatán $(H=0.69$, g. I. $=7, P>0.05)$. No se encontraron diferencias significativas para la frecuencia de ocurrencia de los tres sitios entre ambos ríos $\left(X^{2}=3.00\right.$, g. I. $\left.=1, P=0.11\right)$.

Solamente se lograron identificar dos lugares como áreas de descanso en el segundo transecto del río Zimatán, lugar conocido como "La Escalera" donde se encuentran grandes rocas, pozas, raíces expuestas y cobertura vegetal, aquí se encontraron huellas, excretas y echaderos en donde se pudieron localizar algunos pelos de nutria y restos de su alimento. También fueron encontrados rastros de otros mamíferos tales como huellas y excretas, destacando la presencia del mapache (Procyon lotor) en todos los transectos 
para los dos ríos, aunque con diferente intensidad; así como la presencia de grandes felinos como puma (Puma concolor) y jaguar (Panthera onca; Tabla 2).

\section{Discusión}

Los rastros acopiados durante el muestreo fueron mayores durante la temporada seca y principios de la temporada de lluvia, tanto para la nutria como para los otros mamíferos. Esto puede deberse a que durante la época de lluvias el cauce de ambos ríos aumenta considerablemente eliminando los rastros, además de que la mayoría de las excretas eran lavadas y llevadas al río, perdiendo evidencia de la presencia de estos animales. También se debe considerar que las nutrias pueden defecar dentro del agua, sobre todo cuando tienen crías, probablemente para evitar olores que atraería a depredadores (Melquist y Hornocker 1983; Quadros y Monteiro-Filho 2002); por lo que se debe ser conservador en la interpretación de los resultados ya que tal vez los sitios sean utilizados más de lo que se puede observar por los métodos indirectos.

Aunque estadísticamente no se encontraron diferencias significativas entre las excretas encontradas entre ambos ríos. El mayor número de rastros fueron encontrados en el río Zimatán. Éste presenta las mejores condiciones, tales como la presencia de grandes bloques rocosos, profundidad con corrientes rápidas y con pozas de aproximadamente 1 $\mathrm{m}$ de profundidad, zonas de arena con gran cobertura vegetal, la cual es proporcionada principalmente por las especies del género Acacia y Mimosa, así como lugares apropiados para las madrigueras (Gallo-Reynoso 1989) como se pudo constatar en el segundo transecto al encontrar madrigueras bajo las raíces de los árboles y entre las rocas. Donde no se encontró rastro alguno fue en el último transecto donde se practica el ecoturismo, sobre todo en la parte baja denominado "El Chorro", donde también se encontró ganado, además la mayoría de los habitantes practican la cacería. En un estudio realizado en Brasil, Pardini y Trajano (1999) encontraron que la elección de los lugares como áreas de descanso o de marcaje son al azar y que no existe preferencia por algún sitio en especial, la perturbación del bosque y la presencia humana no influyo en la elección aunque pudieron notar que prefieren los sitios más altos de los ríos. Sin embargo en este río se pudo observar que las nutrias evitan los lugares con actividad antropogénica.

\begin{tabular}{|c|c|c|c|c|c|c|c|c|c|c|}
\hline \multicolumn{8}{|c|}{ Río Copalita } & \multicolumn{3}{|c|}{ Río Zimatán } \\
\hline Tierra & & Roca & & Troncos & & Tierra & & Rocas & & Troncos \\
\hline \multirow[t]{2}{*}{ Transeto } & $\mathrm{N}$ & Transecto & $\mathrm{N}$ & Transecto & N & Transecto & $\mathrm{N}$ & Transecto & $\mathrm{N}$ & Transecto $\mathrm{N}$ \\
\hline & & 1 & 38 & & & 1 & 8 & 1 & 180 & \\
\hline \multirow[t]{2}{*}{2} & 2 & 2 & 75 & 2 & 2 & & & 2 & 295 & \\
\hline & & 3 & 65 & 3 & 4 & & & 3 & 133 & \\
\hline Total & 2 & Total & 178 & Total & 6 & Total & 8 & Total & 608 & Total \\
\hline
\end{tabular}

Tabla 1. Número de en los diferentes sitios
de cada nun de cada uno de los
transectos de muestreo realizados del mes de julio de 1999 al mes en los ríos Copalita Zimatán en el estado de Daxaca.

con preferencia en el segundo sitio. Este río es más largo y su cuenca ha sido alterada por lo que en época de lluvia el agua es de color café intenso debido al arrastre de origen terrígeno por erosión de la parte alta de la cuenca. No es común encontrar rocas mayores a los $2 \mathrm{~m}$, aunque se tienen algunas paredes de aproximadamente $5 \mathrm{~m}$ de altura con grietas donde se encontraron excretas durante la época seca, porque en la época de lluvia se torna imposible el acceso a las paredes. Sin embargo en las zonas adyacentes de arena se encontraron huellas y excretas sobre los troncos caídos principalmente de Ceiba pentandra. El primer transecto (un hábitat altamente perturbado) donde se esperaría no encontrar nada puesto que se extrae material para construcción, fue uno de los sitios con un número considerable de rastros encontrando 51 excretas, con esto para este río se confirma lo encontrado por Pardini y Trajano (1999).

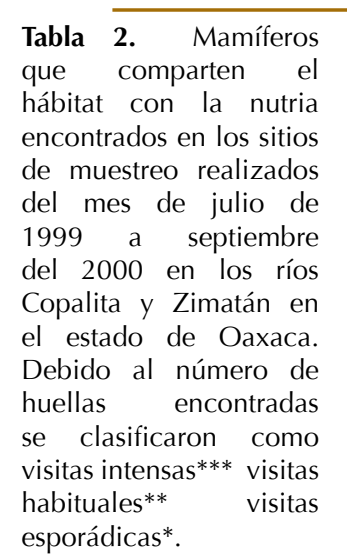

Sitio 1 Procyon lotor *** Dasypus novemcinctus* Odocoileus virginianus Conepatus mesoleucus ${ }^{* * *}$ Sitio 1 Procyon lotor** Herpailurus yagouaround Conepatus mesoleucus*
Río Copalita

Sitio 2

Procyon lotor ${ }^{* * *}$ Conepatus mesoleucus*

Spilogale putorius*

Bassiriscus astatus *

Río Zimatán

Sitio 2

Procyon lotor* Conepatus mesoleucus Dasypus novemcinctus* ${ }^{*}$ Panthera onca*
Sitio Procyon lotor ** Conepatus mesoleucus* Dasypus novemcinctus ${ }^{* *}$ Urocyon cinereoargenteus**

Sitio 3

Procyon lotor* Puma concolor Nasua narica*

Aunque no se encontró diferencia significativa en la frecuencia de uso entre los dos ríos por parte de la nutria, si se observó un mayor uso hacia el río Zimatán particularmente en el segundo transecto. Es aquí donde también se encontraron las dos madrigueras por lo que se podría considerar que los movimientos de la nutria son muy cercanos a su madriguera, quizá porque a diferencia de otros carnívoros terrestres. La nutria hace un uso restringido de su ámbito dentro del medio ambiente, utilizándolo linealmente, es decir se limita al río y a sus márgenes siendo importante para las nutrias el marcaje de su territorio por medio de secreciones (López-Martín et al. 1998; Melquist y Hornocker 1983).

En los lugares donde se encontraron pocos rastros, se podría reflejar una actividad menor de su territorio esto debido probablemente a las condiciones climáticas y densidad poblacional (Quadros y Monteiro-Filhio 2002). Tomando en cuenta el modelo de GalloReynoso (1996) se consideró que para el río Copalita se estima una abundancia relativa de 0.41 nutrias $/ \mathrm{km}$ y para el río Zimatán 1.09 nutrias $/ \mathrm{km}$ (Casariego-Madorell et al. 2008). Esto explicaría la mayor frecuencia de uso en el río Zimatán, ya que serían más los individuos que marcan y protegen su territorio.

En el río Copalita, aunque no se encontraron tantos rastros como en el Zimatán, éstos fueron constantes es decir siempre se encontraron rastros para los tres transectos aunque 
La presencia de otros organismos en los sitios frecuentes de la nutria como la zorra (Urocyon cinereoargenteus) y el jaguaroundi (Puma yaguarondi) probablemente es debido al olor que despiden las excretas de las nutrias por el alimento a base de crustáceos y peces, esto ha sido mencionado por Melquist y Hornocker (1983) encontrando que los mapaches visitan con frecuencia el territorio de la nutria. Otros animales encontrados fueron venado cola blanca (Odocoileus virginianus), coatí (Nasua narica), cacomixtle (Bassariscus astatus), armadillo (Dasypus novemcinctus), y grandes depredadores tales como el puma y el jaguar, que por la cercanía al río de los sitios utilizados por la nutria, éstos se aproximan esporádicamente, ya sea por curiosidad o por coincidencia en el recorrido dentro de su territorio.

Las áreas prioritarias de uso para la nutria neotropical son aquellas que presentan una extensa cobertura vegetal y sitios propicios para la construcción de madrigueras. Su presencia es constante en las áreas rocosas en las playas a la orilla de los ríos y en los troncos caídos. La importancia de las cavidades que se forman bajo las raíces de los árboles y grietas en las rocas, radica en que son sitios visitados frecuentemente como refugio, descanso o para la alimentación tanto por la nutria como por otras especies ya sean depredadores como los felinos y los canidos, y competidores como los prociónidos, entre otros. Esto resalta aún más la importancia que tiene el conservar áreas como los ríos Copalita y Zimatán en Oaxaca, ya que no únicamente cuentan con franjas de bosque tropical caducifolio en buen estado de conservación, que es considerado uno de los ecosistemas terrestres más vulnerables del mundo; sino que además cuenta con especies como la nutria la cual juega un papel importante dentro de dicho ecosistema ya que es un bioindicador en la evaluación de la calidad de ambientes acuáticos.

Agradecimientos

Se agradece el apoyo financiero y logístico del Fondo Mundial para la Conservación de la Naturaleza (WWF). Sociedad para el Estudios de los Recursos Bióticos de Oaxaca (SERBO). Denver Zoological Foundation. Idea Wild, Inc. A B. Miller y G. Ceballos por todo su apoyo y a E. Gamboa-Mijangos por sus comentarios sugeridos al texto.

Literatura citada

Alho, C. J. R., T. E. Lacher, JR., Y H. C. Goncalves. 1988. Environmental degradation in the Pantanal ecosystem. Bioscience 38:164-171.

Alfaro Espinosa, A. M. 2000. Cacería de subsistencia en tres comunidades de la costa de Oaxaca. Tesina. Centro interdisciplinario de Investigación para el Desarrollo Integral Regional, Unidad Oaxaca. Oaxaca, México.

Aranda, M. 2000. Huellas y rastros de los mamíferos grandes y medianos de México. Comisión Nacional para el Conocimiento y uso de la Biodiversidad. Instituto de Ecología A. C. Xalapa, México.

Arita, H. T., y M. Aranda. 1987. Técnicas para el estudio y clasificación de los pelos. Instituto Nacional de Investigaciones sobre Recursos Bióticos. Xalapa, México.

Casariego-Madorelt, M. A. 2004. Abundancia relativa y hábitos alimentarios de la nutria de río (Lontra longicaudis annectens) en la costa de Oaxaca, México. Tesis de Maestría. Facultad de Ciencias. Universidad Nacional Autónoma de México. Ciudad de México, México.

Casariego-Madorell, M. A., R. List, y G. Ceballos. 2008. Tamaño poblacional y alimentación de la nutria de río (Lontra longicaudis annectens) en la costa de Oaxaca, México. Acta Zoológica Mexicana (n. s.) 24:179-200.

Ceballos, G., y A. García. 1995. Conserving neotropical biodiversity: the role of dry forests in western Mexico. Conservation Biology 9:1349-1356.

Chehebár, C. 1991. Searching for the giant otter in northeastern Argentina. IUCN Otter Specialist Group Bulletin 6:17-18.

Contreras, F. 1994. Manual de técnicas hidrológicas. Primera edición, editorial Trillas. Ciudad de México, México.

Franco-lópez, J., G. Agüero, A. Cruz, A. Rocha, N. Navarrete, G. Flores, E. Kato, S. Sanchéz, L. Aabarca, C. M. Bedia, y E. I. Winfield. 1985. Manual de Ecología. Primera edición, editorial Trillas. Ciudad de México, México.

Gallo-Reynoso, J. P. 1986. Otters in Mexico. Journal of the Otter Trust 1:19-24

Gallo-Rernoso, J. P. 1989. Distribución y estado actual de la nutria o perro de agua (Lutra longicaudis annectens Major, 1897) en la Sierra Madre del sur, México. Tesis de Maestría, Facultad de Ciencias. Universidad Nacional Autónoma de México. Ciudad de México, México.

Gallo-Reynoso, J. P. 1996. Distribution of the neotropical river otter (Lutra longicaudis annectens MAJOR, 1897) in the rio Yaqui, Sonora, Mexico. IUCN Otter Specialist Group Bulletin 13:27-31.

García, E. 1981. Modificaciones al sistema de clasificación climática de Köppen. Offset Larios. Ciudad de México, México.

García, G., S. Salas, L. Schibl, R. Aguilar, S. Acosta, y A. Salazar. 1992. Análisis de la vegetación y usos actuales del suelo en el estado de Oaxaca. Fase I (Costa y Sierra sur). Informe técnico SERBO A. C. Oaxaca, México

Gori, M., G. M. Carpaneto, y P. Ottino. 2003. Spatial distribution and diet of the Neotropical otter Lontra longicaudis in the Ibera lake (northern Argentina). Acta Theriologica 48:495-504.

Helder, J., Y K. De Andrade. 1997. Food and feeding habits of the neotropical river otter Lontra longicaudis (Carnivora, Mustelidae). Mammalia 61:193-203.

Hernández, H. A. 1992. Los carnívoros y sus perspectivas de conservación en las áreas protegidas de México. Acta Zoológica Mexicana (n. s.) 54:1-23.

INEGI. 1988. Carta de efectos climáticos. Juchitán. ED15-10D-15-1. Instituto Nacional de Estadística. Geografía e Informática. Ciudad de México, México.

JANZEN, D. H. 1988. Tropical dry forest: the most endangered major tropical ecosystems. Pp. 130-137 in Biodiversity (Wilson, E. O. ed.). National Academy Press. Washington, EE.UU.

LLord, D. R. 1992. Pollution and freshwater fish. A. Buckland Foundation Book. Oxford EE.UU.

López - Martín, J. M., J. Jimenez, y J. Ruíz Olmo. 1998. Caracterización y uso del hábitat de la nutria Lutra lutra (Linné 1758) en un río de carácter mediterráneo. Galemys 10:175-190. 
Macías-Sánchez, S. S., y M. Aranda. 1999. Análisis de la alimentación de la nutria Lontra longicaudis (Mammalia: Carnivora) en un sector del río Los Pescados, Veracruz, México. Acta Zoológica Mexicana (n. s.) 76:49-57.

Macías-Sánchez, S. S. 2003. Evaluación del hábitat de la nutria neotropical (Lontra longicaudis, Olfers 1818) en dos ríos de la zona centro del estado de Veracruz, México. Tesis de Maestria. Instituto de Ecología, A. C. Xalapa, México.

Melquist, W. E. 1983. Latin America's four species of otters: A review of current status and research. Unpublished Report. University of Idaho. Moscow, EE.UU.

Melquist, W. E., Y M. G. Hornocker. 1983. Ecology of river otters in west central Idaho. Journal Wildlife Monographs 83:1-60.

Melquist, W. E., y A. E. Dronkert. 1987. River otter. Pp. 627-641 in Wild Furbearer Management and Conservation in North America (Novak, M., J. A. Baker., M. E. Obbard, y B. Malloch. eds.). Ministry of Natural Resources. Ontario, Canadá.

Orozco-Meyer, A. 1998. Tendencia de la distribución y abundancia de la nutria de río (Lontra longicaudis annectens Major, 1897), en la ribera del río Hondo, Quintana Roo, México. Tesis de Licenciatura. Instituto Tecnológico de Chetumal. Chetumal, México.

Pardini, R., y E. Trajano. 1999. Use of shelters by the neotropical river otter (Lontra longicaudis) in an Atlantic forest stream, southeastern Brazil. Journal of Mammalogy 80:600-610.

Parera, A. 1992. Dieta de Lutra longicaudis en la laguna Iberá, Provincia de Corrientes, Argentina. V Reunión de Especialistas en Mamíferos Acuáticos de América del Sur. Libro de Resúmenes. Buenos Aires, Argentina.

Parera, A. 1996. Las nutrias verdaderas de la Argentina. Fundación Vida Silvestre Argentina. Boletín técnico 21:13-20.

QuAdRos, J., Y E. L. Monteiro - Filho. 2002. Sprainting sites of the neotopical otter Lontra longicaudis in an Atlantic forest area of Southern Brazil. Journal of Neotropical Mammalogy 9:39-46.

Ruíz-Olmo, J., D. SaAvedra, y J. Jiménez. 2000. Testing the surveys and visual and track censuses of Eurasian otters (Lutra lutra). Zoological Journal of the Linnean Society of London 253:359-369.

Ruíz-Olmo, J., J. Jiménez., S. Palazón., M. Delibes, C. Bravo, y F. Bueno. 1998. Factores que han determinado la situación actual de las poblaciones de nutria y propuestas de gestión. Pp. 223-242 in La nutria en España ante el horizonte 2000 (Ruiz-Olmo, J., y M. Delibes, eds.). Sociedad Española para la Conservación y Estudio de los Mamíferos (SECEM) Grupo Nutria. Barcelona-Sevilla-Málaga, España.

Rzedowski, J. 1978. Vegetación de México. Editorial Limusa. Ciudad de México, México.

Salas-Morales, S. H. 2002. Relación entre la heterogeneidad ambiental y la variabilidad estructural de las selvas tropicales secas de la costa de Oaxaca, México. Tesis de Maestría. Facultad de Ciencias, Universidad Nacional Autónoma de México. Ciudad de México, México.

Salas, S., E. Torres, A. Gonź́lez, LCHibu, H. Morales, y M. Cerón. 1996. Análisis de la vegetación y uso actual del suelo en el estado de Oaxaca, Fase IV (Istmo). Informe Técnico. SERBO. A. C. Oaxaca, México. (No publicado).
SEMARNAT. 2010. NORMA Oficial Mexicana NOM-059-SEMARNAT-2010, Protección ambiental-Especies nativas de México de flora y fauna silvestres-Categorías de riesgo y especificaciones para su inclusión, exclusión o cambio-Lista de especies en riesgo. Diario Oficial de la Federación. Jueves 30 de Diciembre de 2010.

Spínola, R., Y C. Vaughan. 1995. Dieta de la nutria neotropical (Lutra longicaudis) en la estación biológica La Selva, Costa Rica. Vida Silvestre Neotropical 4:125-132.

Stalb, E., y C. Schenck. 1994. Lobo de río. Zoologische Gessllschaft frankfort bedrohte trerwelt - schweizerische Kredition Stalt deutschland AG:14-15.

Zar, J. H. 1999. Biostatistical Analysis. Prentice-Hall. Upper Saddle River, EE.UU.
Sometido: 17 de mayo de 2013 Revisado: 20 de julio de 2013 Aceptado: 26 de noviembre de 2013 Editor asociado: Juan Pablo Gallo Diseño gráfico editorial: Gerardo Hernández 
